\title{
Associative Hebbian Synaptic Plasticity in Primate Visual Cortex
}

\author{
Shiyong Huang, ${ }^{1}$ Carlos Rozas, ${ }^{1}$ Mario Treviño, ${ }^{1}$ Jessica Contreras, ${ }^{1}$ Sunggu Yang, ${ }^{1}$ Lihua Song, ${ }^{1}$ Takashi Yoshioka, ${ }^{1}$ \\ Hey-Kyoung Lee, ${ }^{1,2}$ and Alfredo Kirkwood ${ }^{1,2}$ \\ ${ }^{1}$ The Mind/Brain Institute, Johns Hopkins University, Baltimore, Maryland 21218 and ${ }^{2}$ Department of Neuroscience, Johns Hopkins University, Baltimore, \\ Maryland 21205
}

In primates, the functional connectivity of adult primary visual cortex is susceptible to be modified by sensory training during perceptual learning. It is widely held that this type of neural plasticity might involve mechanisms like long-term potentiation (LTP) and long-term depression (LTD). NMDAR-dependent forms of LTP and LTD are particularly attractive because in rodents they can be induced in a Hebbian manner by near coincidental presynaptic and postsynaptic firing, in a paradigm termed spike timing-dependent plasticity (STDP). These fundamental properties of LTP and LTD, Hebbian induction and NMDAR dependence, have not been examined in primate cortex. Here we demonstrate these properties in the primary visual cortex of the rhesus macaque (Macaca mulatta), and also show that, like in rodents, STDP is gated by neuromodulators. These findings indicate that the cellular principles governing cortical plasticity are conserved across mammalian species, further validating the use of rodents as a model system.

Key words: LTD; LTP; monkey; STDP

\section{Introduction}

The functional connectivity of the visual cortex in primates is highly modifiable by visual experience. The plasticity of the visual cortex manifests strongly at an early postnatal age when abnormal or reduced visual experience can result in lasting impairment of visual function, including amblyopia in humans (Daw, 1998). In adults, the reduction in detection threshold associated with several forms of training-induced perceptual learning also results from changes in the primary visual cortex (Gilbert et al., 2009). It is widely, but not universally, considered that these modifications of cortical connectivity depend crucially on activity-dependent forms of synaptic plasticity like long-term potentiation (LTP) and long-term depression (LTD) (Feldman, 2009).

The rules and mechanism of induction of both LTP and LTD have been extensively studied in rodents. In the superficial layers of mouse and rat visual cortex the induction and expression of synaptic plasticity in pyramidal cells is postsynaptic and depends on the activation of NMDARs. Importantly, its induction is Hebbian, that is, LTP results when presynaptic and postsynaptic activity correlates, and LTD when it does not (Smith et al., 2009). Moreover, plasticity can be induced in an associative manner by pairing near coincidental presynaptic and postsynaptic action potential in a paradigm termed spike timing-dependent plasticity

Received March 11, 2014; revised April 21, 2014; accepted April 27, 2014.

Author contributions: A.K. designed research; S.H., C.R., M.T., J.C., S.Y., L.S., and T.Y. performed research; S.H.,

C.R., M.T., S.Y., and L.S. analyzed data; H.-K.L., and A.K. wrote the paper.

This work is supported by grants R01 EY012124 and R01AG034606 (A.K.) and R01 EY014882 (H.-K.L.)

The authors declare no competing financial interests.

Correspondence should be addressed to Alfredo Kirkwood, The Mind/Brain Institute, Johns Hopkins University, 350 Dunning Hall, 3400 N. Charles St., Baltimore, MD 21218. E-mail: Kirkwood@jhu.edu.

DOI:10.1523/JNEUROSCI.0983-14.2014

Copyright $\odot 2014$ the authors $\quad 0270-6474 / 14 / 347575-05 \$ 15.00 / 0$
(STDP), which is widely considered a plausible model for synaptic modification occurring in vivo (Caporale and Dan, 2008; Richards et al., 2010; but see Lisman and Spruston, 2005). Although LTP and LTD have been demonstrated in human cortical slices (Chen et al., 1996), Hebbian associativity, the fundamental feature that makes NMDAR-dependent plasticity an attractive mechanism to subserve cortical learning, has not been examined in primate visual cortex.

It is not a foregone conclusion that the principles uncovered in rodent visual cortex are necessarily applicable to primate visual cortex. The rules and mechanism for the induction of LTP and LTD can vary widely across synapses, and the primary visual cortex is highly specialized anatomically, exhibiting a unique pattern of lamination and compartmentalization in functional modules that largely departs from the general connectivity pattern of isocortex. Prompted by these considerations we examined the induction of synaptic plasticity in the supragranular layers of visual cortex of adult macaque monkeys. We found that it depends on NMDARs, it is Hebbian, and it can be induced in an associative manner with STDP paradigms.

\section{Materials and Methods}

The experiments were performed in visual cortical slices (300-400 $\mu \mathrm{m})$ from seven 3- to-5-year-old rhesus monkeys (Macaca mulatta) of either sex. The tissue ( $\mathrm{a} \sim 25 \mathrm{~mm}^{3}$ block from the binocular zone of area 17) was extracted during euthanasia after sedation with ketamine $(10 \mathrm{mg} / \mathrm{kg}$, i.m.) and deep anesthesia with pentobarbital $(25 \mathrm{mg} / \mathrm{kg})$ according to protocols approved by the Johns Hopkins Institutional Animal Care and Use Committee.

The slices were cut in ice-cold dissection buffer containing the following (in mM): 212.7 sucrose, $5 \mathrm{KCl}, 1.25 \mathrm{NaH}_{2} \mathrm{PO}_{4}, 10 \mathrm{MgCl}_{2}, 0.5 \mathrm{CaCl}_{2}$, $26 \mathrm{NaHCO}_{3}$, and 10 dextrose, bubbled with $95 \% \mathrm{O}_{2} / 5 \% \mathrm{CO}_{2}, \mathrm{pH} 7.4$, and after $1 \mathrm{~h}$ they transferred to artificial CSF (ACSF) of similar compo- 
sition except that sucrose is replaced by $124 \mathrm{~mm} \mathrm{NaCl}, \mathrm{MgCl}_{2}$ is lowered to $1 \mathrm{~mm}$, and $\mathrm{CaCl}_{2}$ is raised to $2 \mathrm{~mm}$. Baseline responses $(0.05 \mathrm{~Hz})$ were evoked with $0.2 \mathrm{~ms}$ pulses delivered through two concentric bipolar stimulating electrodes (125 $\mu \mathrm{m}$ diameter; FHC) placed $\sim 900 \mu \mathrm{m}$ apart in the upper border of the line of Gennari. Input independence was confirmed by linear summation and the absence of paired-pulse interactions.

Visualized whole-cell current-clamp recordings were made from layer III regular-spiking pyramidal cells with glass pipettes (4-6 M $\Omega$ ) filled with the following (in $\mathrm{mm}$ ): $130(\mathrm{~K})$ gluconate, $10 \mathrm{KCl}, 0.2 \mathrm{EGTA}, 10$ HEPES, $4(\mathrm{Mg})$ ATP, $0.5(\mathrm{Na}) \mathrm{GTP}$, and 10 (Na)Phosphocreatine, $\mathrm{pH}$ adjusted to 7.25 , with $\mathrm{KOH}, 280-290$ mOsm. Only cells with stable membrane potentials $<-65 \mathrm{mV}$, series resistance $<20 \mathrm{M} \Omega$, and input resistance $>100 \mathrm{M} \Omega$ were studied. Synaptic responses $(4-6 \mathrm{mV})$ were filtered at $2 \mathrm{kHz}$, digitized at $5 \mathrm{kHz}$, and quantified as the initial slope of the EPSP using Igor Pro (WaveMetrics).

Layer III field potentials (FPs) were recorded in $400 \mu \mathrm{m}$ slices with ASCF-filled pipettes with stimulus intensities that evoked FPs approximately half of the maximal amplitude $(\sim 0.7-1.5 \mathrm{mV})$. To induce LTP we used theta burst stimulation (TBS) consisting of four epochs $(0.1 \mathrm{~Hz})$ of 10 bursts (four stimuli at $100 \mathrm{~Hz}$ ) delivered at $5 \mathrm{~Hz}$. To induce LTD we used

low-frequency stimulation (LFS; 900 pulses at $1 \mathrm{~Hz}$ ).

Pairing-induced synaptic plasticity was performed under voltageclamp as described previously (Huang et al., 2012) and consisted of 150 epochs $(0.75 \mathrm{~Hz})$ during which the holding potential $\left(V_{\mathrm{h}}\right)$ alternated between two target values dwelling $666 \mathrm{~ms}$ in each value $(-70$ and -10 $\mathrm{mV}$ for LTP, or -70 and $-40 \mathrm{mV}$ for LTD). In each pairing epoch the synaptic stimulation pulse was delivered $100 \mathrm{~ms}$ after the onset of depolarization. STDP was induced by pairing presynaptic stimulation in one or the two pathways with a burst of four action potentials $(100 \mathrm{~Hz})$ evoked by passing suprathreshold depolarizing current steps through the recording electrode ( $\sim 1 \mathrm{nA}, 2 \mathrm{~ms}$ ) as described in rodents (Seol et al., 2007). Associative pairing consisted of 200 pairing epochs delivered at 1 $\mathrm{Hz}$.

Immunoblot analysis was done as previously described (Goel et al., 2011). In brief, visual cortex samples were homogenized and membrane fraction was isolated. Samples were resuspended in gel sample buffer and loaded onto SDS-PAGE gels ( $20 \mu \mathrm{g} / \mathrm{lane})$. After transfer onto polyvinyl difluoride membranes (Immobilon; Millipore), the blots were processed by simultaneously incubating in either GluA1-S831 or GluA1-S845 phospho-specific antibody (rabbit polyclonal affinity purified in-house) and GluA1-C terminal antibody (mouse monoclonal: sc-55509; Santa Cruz Biotechnology) followed by simultaneous incubation in secondary antibodies linked to anti-rabbit Cy3 and anti-mouse Cy5 (ECLplex; GE Health). Blots were imaged using a Typhoon scanner (GE Health), and quantified using Image Quant-TL (GE Health). Signal from phosphospecific antibodies was divided by GluA1-C terminal antibody signal then normalized to the average control value for comparison across blots.

Statistical analysis. Group plots are presented as average \pm SEM. The magnitude of plasticity was taken as the average of the last $10 \mathrm{~min}$ of recording, beginning $20 \mathrm{~min}$ after conditioning stimulation. The significance of LTP and LTD was assessed using the paired Student's $t$ test. Other comparisons were done using Student's $t$ test or the ANOVA test.

\section{Results}

We investigated the induction of LTP and LTD in acute visual cortical slices from adult primates using three different, yet complementary, sets of conditioning paradigms previously tested in rodents. These approaches were as follows: (1) frequencydependent protocols with extracellular recordings, which produce minimal interference on intracellular processes; (2) pairing protocols, the most effective procedures to induce LTP and LTD; and (3) STDP protocols, which are widely although not universally regarded as a better approximation for the induction of plasticity in vivo. To better compare findings in rodents we studied the synaptic responses in layer III evoked by layer IV stimu-
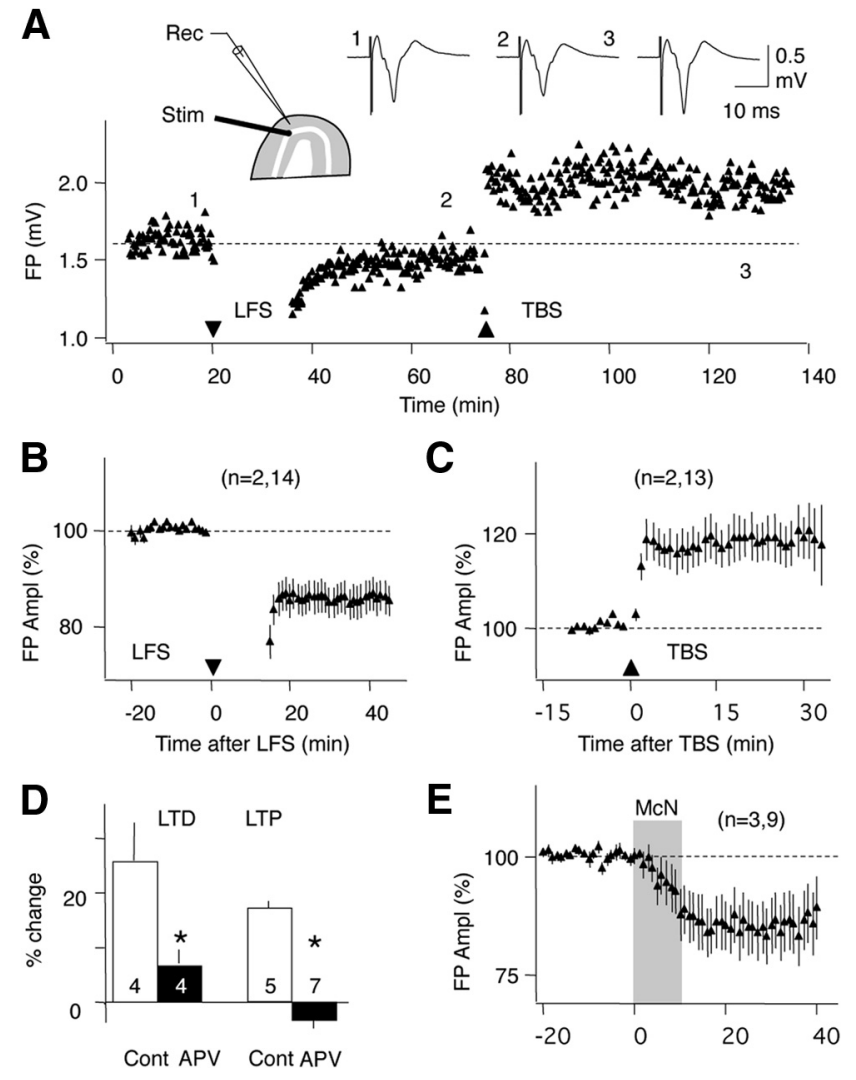

Figure 1. Bidirectional synaptic plasticity of the supragranular FPs evoked by layer IV stimulation. $A$, Example of sequential induction of LTD with LFS and LTP with TBS. FPs were recorded in the upper supragranular layers, and stimulation was applied at the upper border of the line of $G$ Gnnari. Traces are averages of four consecutive FPs recorded at the indicated times. $\boldsymbol{B}$, Average induction of LTD by LFS. C, Average induction of LTP by TBS. D, LTP and LTD depend on NMDARs. Left, Average LTP (measured 30 min after TBS) induced in $100 \mu \mathrm{m} \mathrm{APV} \mathrm{(black} \mathrm{bar)} \mathrm{and} \mathrm{in} \mathrm{control}$ ACSF (Cont; white bar). Right, Average LTD (30 min after LFS) induced in $100 \mu \mathrm{m}$ APV (black bar) and in control ACSF (white bar). E, LTD can also be induced by bath application of the M1 muscarinic agonist $\mathrm{McN}$ (gray bar; $10 \mu \mathrm{M}, 10 \mathrm{~min}$ ) during baseline stimulation with paired pulse (50 ms interval). Number of monkeys and experiments is indicated in parentheses.

lation, and we used the exact same conditioning protocols and ACSF composition as in previous studies.

\section{Bidirectional synaptic plasticity in primate visual cortex}

First, we examined the induction of bidirectional synaptic changes with frequency-dependent paradigms, which in rodents have been extensively tested in multiple cortical regions. We used LFS ( $1 \mathrm{~Hz}, 15 \mathrm{~min}$ ) to induce LTD and high-frequency stimulation (TBS) to induce LTP of the local FPs recorded in layer III (see Materials and Methods). Of particular interest was the induction of LTD with LFS, a conditioning paradigm that in rats is effective across all ages, but in mice only in juveniles (Jiang et al., 2007). The results indicated that in visual cortical slices from adult monkey, conditioning with LFS induces LTD $(85.9 \pm 3.5 \%$ of baseline measured 30 min after LFS, $n=2$ monkeys, 14 slices; paired $t$ test: $p<0.001$; Fig. $1 A, B)$. In two cases, one of them shown in Figure $1 A$, we confirmed that LFS-induced LTD can be de-depressed by high-frequency stimulation in the form of TBS (Fig. $1 A$ ). On the other hand, in naive slices conditioning with TBS induces clear LTP $(117.8 \pm 7.0 \%$ of baseline measured $30 \mathrm{~min}$ after LFS, $n=$ 2,13; paired $t$ test: $p=0.002$; Fig. $1 A, C)$. In rodents and cats, the induction of cortical LTP and LTD with frequency-dependent paradigms requires the activation of NMDARs. We confirmed 
A

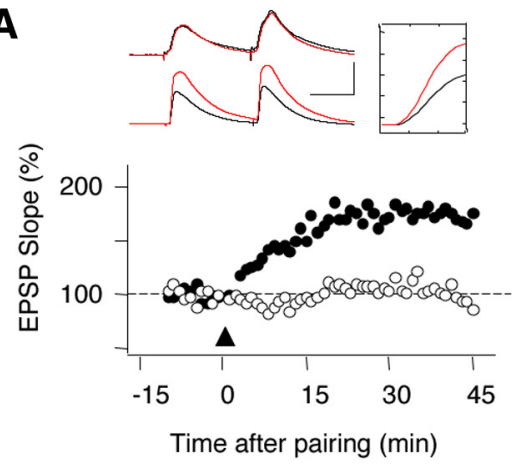

C

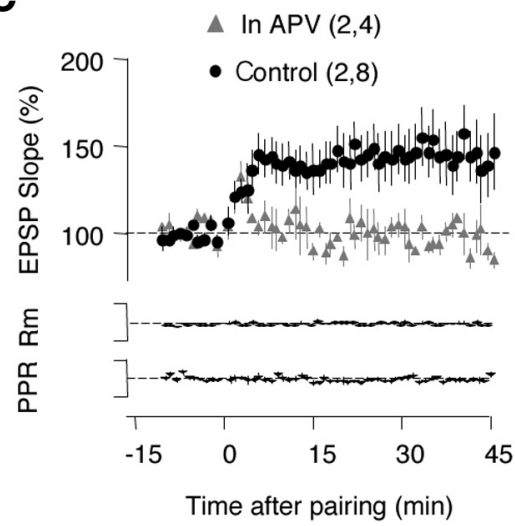

B
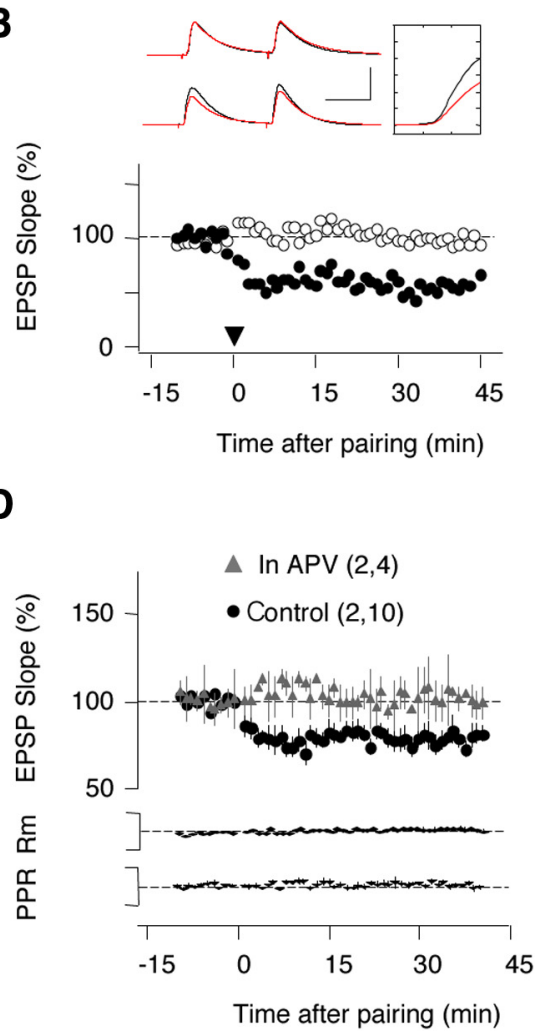

Figure 2. Hebbian induction of LTP and LTD in layer III pyramidal cells. $A, B$, Example two-pathway experiments showing homosynaptic pairing-induced LTP $\left(A, V_{\mathrm{m}}: 0 \mathrm{mV}\right)$ and LTD $\left(\boldsymbol{B}, V_{\mathrm{m}}:-40 \mathrm{mV}\right)$. Synaptic changes were induced in the stimulated pathway (filled circles), but not in the nonstimulated pathway (open circles). Traces on top are averages of 10 consecutive responses recorded before (black) and $30 \mathrm{~min}$ after pairing (red). Calibration: $A, B, 10 \mathrm{~ms}, 5 \mathrm{mV}$. Inset, Each tick represents $1 \mathrm{~ms}, 1$ $\mathrm{mV}$. $\boldsymbol{C}, \boldsymbol{D}$, NMDAR activation is required for pairing-induced LTP $(\boldsymbol{C})$ and for pairing-induced LTD (D). Top graphs, Changes in the EPSP induced by pairing in control ACSF (filled circles) and in $100 \mu \mathrm{m}$ APV (gray triangles). Bottom graphs, PPR and input resistance $\left(R_{\text {in }}\right)$ for the control experiments (both expressed as percentage of baseline).

that this is also the case in the slices of adult primates: addition of the NMDAR antagonist APV $(100 \mu \mathrm{M})$ blocked the induction of both LTD (Control: $74.9 \pm 7.0, n=4$; APV: $92.3 \pm 2.6, n=4 ; t$ test: $p=0.024$; Fig $1 D$ ) and LTP (Control: $117.4 \pm 1.4, n=5$; APV: $96.9 \pm 1.5, n=7$; $t$ test: $p<0.001$; Fig $1 D)$. A final issue of interest was the cholinergic regulation of LTD. In rodents (rats and mice), stimulation of M1 muscarinic receptors facilitates the induction of NMDAR-dependent LTD to the extent that bath application of agonist allows the induction of LTD even with a baseline type of stimulation (paired-pulse stimulation with $50 \mathrm{~ms}$ interval delivered at $0.07 \mathrm{~Hz}$; Kirkwood et al., 1999). In contrast, in the visual cortex of the tree shrew, an insectivore that is phylogenetically closer to primates, the same stimulation regime in conjunction with M1 receptor stimulation was reported to promote LTP, not LTD (McCoy et al., 2008). Therefore, we tested in the primate visual cortex the effects of a brief application of the $\mathrm{M} 1$ agonist $\mathrm{McN}(10 \mu \mathrm{M}$ for $10 \mathrm{~min})$, and found that it resulted in robust LTD $(84.9 \pm 5.3 \%$ of baseline, $n=3,9$; paired $t$ test: $p=$ 0.024 ; Fig. $1 E$ ). Altogether the results confirmed the induction of NMDAR-dependent bidirectional plasticity in primate visual cortex, and confirmed the cholinergic facilitation of LTD as previously described in rodents.

Hebbian induction of LTP/LTD in primate visual cortex A fundamental property of NMDAR-dependent plasticity is its Hebbian induction; that is, the level of postsynaptic activity determines the polarity of plasticity. In rodent visual cortical slices, Hebbian plasticity is revealed by showing that presynaptic stimulation results in LTP when paired with strong postsynaptic depolarization, and results in LTD if paired with moderate postsynaptic depolarization (Huang et al., 2012). Therefore, we used a similar pairing approach to determine whether synaptic plasticity is also Hebbian in primates. In these experiments synaptic responses were recorded with whole-cell methods and the pairing consisted of 120 presynaptic pulses delivered at $1 \mathrm{~Hz}$ while holding the postsynaptic potential at $0 \mathrm{mV}$ or $-40 \mathrm{mV}$ to respectively induce LTP or LTD of the EPSP (see Materials and Methods). As shown in Figure 2, pairing with depolarization to 0 $\mathrm{mV}$ induced LTP $(151 \pm 14 \%$ of baseline, $n=2,8$; paired $t$ test: $p=0.01$; Fig. $2 A, C)$ without affecting the paired-pulse ratio (PPR) of the responses (before: $0.963 \pm$ 0.009; after: $0.980 \pm 0.001$; paired $t$ test: $p=$ 0.61 ), an indicator of presynaptic changes. On the other hand, pairing with depolarization to $-40 \mathrm{mV}$ induced LTD (79.4 $\pm 5.6 \%$ of baseline, $n=2,10$; paired $t$ test: $p=0.003$; Fig. $2 B, D$ ) without affecting the PPR (before: $1.017 \pm 0.004$; after: $1.015 \pm 0.019$; paired $t$ test: $p=0.98$ ). We confirmed that pairing-induced plasticity depends on the activation of NMDARs as bath application of the antagonist APV $(100 \mu \mathrm{M})$ blocked the induction of LTP (in APV: $90.9 \pm 9.1 \%, n=$ 2,4 ; $t$ test: $p=0.039$ ) and LTD (in APV: $102.6 \pm 8.3 \%, n=2,4 ; t$ test: $p=0.042)$.

Another fundamental property of synaptic plasticity well documented in rodents is input specificity: only conditioned synapses undergo plastic changes. To test whether this is also the case in primate visual cortex, in a subset of the experiments we recorded from an additional independent pathway, which was not stimulated during the pairing paradigm. The responses of nonconditioned pathway were not affected by the induction of either LTP (conditioned: $159.5 \pm 21.3 \%$; nonconditioned: $102.3 \pm 2.8, n=$ 4 experiments; paired $t$ test: $p=0.03$; example experiment in Fig. $2 A$ ) or LTD (conditioned: $79.6 \pm 7.2 \%$; nonconditioned: $104.4 \pm$ 8.7, $n=7$; paired $t$ test: $p=0.02$; example experiment in Fig. $2 B$ ). Altogether, the results confirmed that primate visual cortex does express postsynaptic forms of LTP and LTD that are Hebbian and input specific.

\section{STDP in primate visual cortex}

Finally, we examined the induction of synaptic plasticity with STDP associative paradigms based on near coincidental presynaptic and postsynaptic firing. In rodents we previously showed that pairing presynaptic activation with postsynaptic bursts of four action potentials at $100 \mathrm{~Hz}$ induces LTP when the postsynaptic bursts are delivered $10 \mathrm{~ms}$ after the presynaptic stimulation (pre-then-post) and LTD if delivered $10 \mathrm{~ms}$ before (post-thenpre), but only when gated by the stimulation of receptors coupled to the G-proteins $\mathrm{G}_{\mathrm{s}}$ and $\mathrm{G}_{\mathrm{q}}$ (Seol et al., 2007; Guo et al., 2012). Similarly, in the primate slices under our standard experimental conditions these STDP pairings (120 epochs at $1 \mathrm{~Hz}$; see also Materials and Methods) did not affect the synaptic responses, 
either when the order was pre-then-post $(100.7 \pm 5.5 \%, n=2,9$; paired $t$ test: $p=$ 0.86 ; Fig. $3 A)$ or the reverse, post-thenpre $(98.6 \pm 4.1 \%, n=2,9$; paired $t$ test: $p=0.96$. Fig. $3 A$ ). Therefore, as shown in Figure 3, $B$ and $C$, we tested the effects of costimulating $\beta$-adrenergic receptors (coupled to $\mathrm{G}_{\mathrm{s}}$ ) and $\alpha 1$-adrenergic receptors (coupled to $\mathrm{G}_{\mathrm{q}}$ ) by a $10 \mathrm{~min}$ bath application of their respective agonists (10 $\mu \mathrm{M}$ isoproterenol and $5 \mu \mathrm{M}$ methoxamine). Under these conditions the spiketiming requirement was enabled such that pre-then-post pairing induced tLTP $(156.4 \pm 16.3 \%, n=3,10$; paired $t$ test: $p=0.006)$, and the post-then-pre paring induced tLTD $(78.7 \pm 7.5 \%, n=3,8$; paired $t$ test: $p=0.038)$. In both cases there were no changes in PPR (PPR before LTD: $0.91 \pm 0.08$, after LTD: $1.02 \pm 0.06$, paired $t$ test: $p=0.16$; PPR before LTP: $1.05 \pm 0.04$, after LTP: $1.02 \pm 0.05$, paired $t$ test: $p=0.46$ ). Thus, the results indicate that in primates, as in rodents, neuromodulators are essential for the gating of visual cortical STDP.

In rodent visual cortex, the phosphorylation of AMPA receptors at specific sites that control receptor trafficking (Huganir and Nicoll, 2013) is crucial for the neuromodulatory control of STDP (Seol et al., 2007). In slices from rodents, the stimulation of $\beta$-adrenergic receptors with isoproterenol promotes LTP by phosphorylating serine 845 in the GluA1 subunit (S845), while stimulation of $\mathrm{M} 1$ cholinergic receptors with McN promote LTD by phosphorylating S845 and the serine 831 site (S831). Therefore, we used immunoblot analysis to examine whether this is also the case in primate visual cortex. As shown in Figure 3D, a 10 min exposure to isoproterenol (Iso:10 $\mu \mathrm{M}$ ) increased phosphorylation of S845 (266.0 $\pm 61.0 \%$ of control, $n=$ $2,7 ; t$ test: $p=0.035)$, but not S831 (95.2 $\pm 6.4 \%$; $t$ test: $p=0.61$ ), while exposure to $\mathrm{McN}(10 \mu \mathrm{M})$ did not increase significantly the phosphorylation of either S845 (148.2 $\pm 21.3 \%, n=2,7, t$ test: $p=$ $0.089)$ or S831 $(100.3 \pm 15.9 \%, t$ test: $p=0.985)$. Methoxamine (Mtx; $5 \mu \mathrm{M}$ ), an agonist for the $\mathrm{G}_{\mathrm{q}}$-coupled $\alpha 1$-adrenergic receptor, did not affect any of these sites (S845: $108.1 \pm 18.1 \%, n=2,4$, $t$ test: $p=0.69$; S831: $88.12 \pm 12.8 \%, t$ test: $p=0.444)$. Thus, as in rodents GluA1-S845 phosphorylation increase accompanies neuromodulation of spike-timing LTP in primates. However, there is some difference in that GluA1-S831 phosphorylation is not altered by any of the neuromodulators in primate cortex.

\section{Discussion}

Essential features of Hebbian synaptic plasticity are that the polarity and magnitude of the synaptic changes are determined by the correlation between presynaptic and postsynaptic activity, and that these changes are confined to conditioned synapses. In this study we showed that the primary visual cortex of primates expresses Hebbian NMDAR-dependent homosynaptic forms of LTP and LTD, which can be induced in an associative manner with STDP paradigms. Thus, the fundamental principles governing the induction of synaptic plasticity, originally characterized in
B
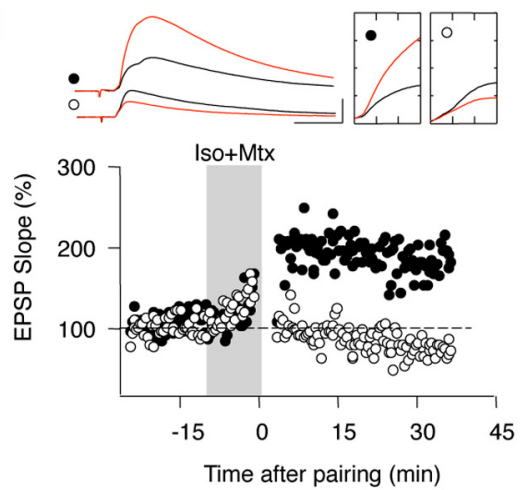

D
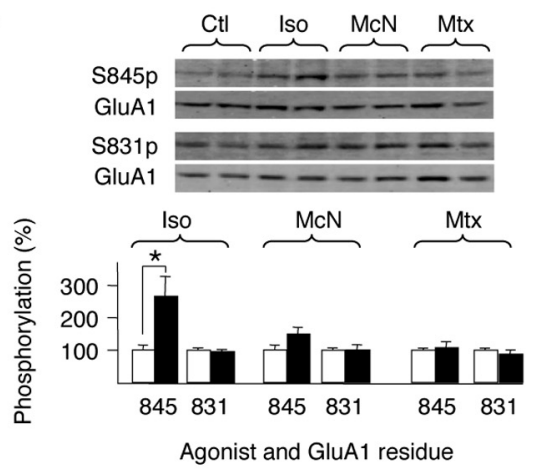

Figure 3. Adrenergic gating of STDP. A, In control ACSF associative spike-timing paradigms induce neither tLTP (filled circles) nor tLTD (open circles). The diagram illustrates the conditioning paradigm (s1: stimulation of input 1, a.p: postsynaptic action potential, s2: stimulation of input 2). B, C, Bath application of agonists for $\beta$ - and $\alpha 1$-adrenergic receptors (isoproterenol, Iso: 10

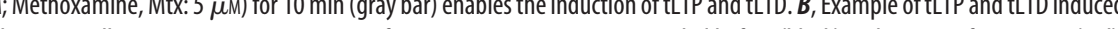
GluA1 phosphorylation (percentage of control; $\mathrm{Ct}$ ) at the residues $\mathrm{S} 845$ and $\mathrm{S} 831$ induced by a 10 min exposure to either $10 \mu \mathrm{m}$ Iso, $10 \mu \mathrm{M} M \mathrm{MN}$, or $5 \mu \mathrm{m}$ Mtx. Example blots are shown on the top.

rats and mice, appear to be conserved across mammalian species, which in turn further validate the use of rodent models for research on the underlying mechanisms.

Although the rules of synaptic plasticity are conserved, the optimal stimulation parameters to recruit it might vary among species. For example, extracellular LFS does not elicit LTD in cortical and hippocampal synapses after puberty in mice (Jiang et al., 2007), but it does in adult rats (Dudek and Bear, 1993) and monkeys (Fig. 1). However, other conditioning paradigms that allow direct control of postsynaptic activity, like pairing (Jiang et al., 2007) and STDP, can effectively induce LTD after puberty in mice (Hardingham et al., 2008). Thus, off-target contingencies associated with extracellular stimulation, like the activation of strong inhibitory GABAergic networks, for example (Jiang et al., 2005), might limit the activation of NMDARs during LFS, impairing the induction of LTD with purely extracellular methods. These considerations warrant that differences detected with extracellular methods need to be confirmed with intracellular methods. Indeed, this inherent limitation of extracellular approaches might account for the observation that in visual cortical slices of an insectivore, the tree shrew, bath application of M1 cholinergic agonists does not promote LTD (McCoy et al., 2008) of extracellularly recorded layer II/III field responses as it does in rodents (Massey et al., 2001) and primates (Fig. 1).

Bidirectional associative synaptic plasticity is an attractive mechanism to subserve the cortical modifications involved in perceptual and motor learning in adults. The development of 
noninvasive approaches to stimulate cortex like transcranial magnetic stimulation (TMS) and transcranial direct current stimulation (TDCS) made it possible to directly induce and study cortical plasticity in humans (Koch, 2013; Schulz et al., 2013; Vallence and Ridding, 2013). A typical application is paired associated stimulation (PAS), in which a response to cortical TMS is modified by repeatedly pairing it with a strong conditional stimulation (Koch, 2013). In this type of paradigm the temporal relationship between the test and conditioning stimulation determines the polarity of plasticity, with LTP-like changes resulting when the test stimulation is followed by the strong stimulation, and in LTD-like changes, resulting when the test is preceded by the strong stimulation. In this context, our demonstration that similar timing rules govern STDP in rodent and primates is highly significant. Notably, in the primate cortical slices, as in rodent slices from many brain regions, the induction of STDP is gated by the action of neuromodulators and the coactivation of $\beta$-adrenergic and $\alpha 1$-adrenergic receptors is sufficient to support bidirectional changes. In rodents, the phosphorylation of AMPA receptors at specific sites by neuromodulators might prime the induction of LTP and LTD with STDP paradigms. We confirmed that in primate slices $\beta$-adrenergic activation increases the phosphorylation of GluA1 serine 845, necessary for both LTP and LTD, but we did not observe any phosphorylation changes induced by agonists that promote LTD. One possibility is that the basal phosphorylation state, which is known to vary among different cortices (Goel et al., 2006) and visual experience (Goel et al., 2006, 2011), may be already higher in primate slices obscuring the detection of further increases. Nevertheless, even if the neuromodulation of associative plasticity differs in some molecular details in primate and rodents, the general rules of its neuromodulatory gating are seemingly comparable. Moreover, such generality dovetails with the observation that associative Hebbian plasticity of excitatory inputs onto inhibitory interneurons, although it is independent of NMDAR activation, it is also gated by neuromodulators (Huang et al., 2013). Thus, we propose that in cortex, although mechanisms might vary, similar rules govern associative plasticity of excitatory connections across different synapses and species.

The demonstration of associative cortical plasticity in humans was not only of great conceptual significance, but also opened the possibility of using noninvasive approaches to modify synaptic strength for therapeutic purposes, including the strengthening of specific pathways to restore/compensate for loss of function, or the weakening of pathways to revert maladaptive changes (Schulz et al., 2013). Our confirmation that neuromodulation of associative plasticity in primate slices operates under rules that are conserved across species can have important consequences for interventions in humans. In rodent visual cortex the activation of GPCRs gate LTP, if linked to $G_{s}$, and gate LTD if linked to $G_{\mathrm{q}}$. This feature can be exploited to potentiate or depress visual cortical synapses in vivo by the conjunction of visual stimulation with ligands targeting specific GPCRs. It is conceivable that a similar approach in humans could be used to enhance the selectivity and effectiveness of plasticity induced with noninvasive approaches.

\section{References}

Caporale N, Dan Y (2008) Spike timing-dependent plasticity: a Hebbian learning rule. Annu Rev Neurosci 31:25-46. CrossRef Medline

Chen WR, Lee S, Kato K, Spencer DD, Shepherd GM, Williamson A (1996)

Long-term modifications of synaptic efficacy in the human inferior and middle temporal cortex. Proc Natl Acad Sci U S A 93:8011-8015. CrossRef Medline

Daw NW (1998) Critical periods and amblyopia. Arch Ophthalmol 116: 502-505. CrossRef Medline

Dudek SM, Bear MF (1993) Bidirectional long-term modification of synaptic effectiveness in the adult and immature hippocampus. J Neurosci 13:2910-2918. Medline

Feldman DE (2009) Synaptic mechanisms for plasticity in neocortex. Annu Rev Neurosci 32:33-55. CrossRef Medline

Gilbert CD, Li W, Piech V (2009) Perceptual learning and adult cortical plasticity. J Physiol 587:2743-2751. CrossRef Medline

Goel A, Jiang B, Xu LW, Song L, Kirkwood A, Lee HK (2006) Cross-modal regulation of synaptic AMPA receptors in primary sensory cortices by visual experience. Nat Neurosci 9:1001-1003. CrossRef Medline

Goel A, Xu LW, Snyder KP, Song L, Goenaga-Vazquez Y, Megill A, Takamiya K, Huganir RL, Lee HK (2011) Phosphorylation of AMPA receptors is required for sensory deprivation-induced homeostatic synaptic plasticity. PLoS One 6:e18264. CrossRef Medline

Guo Y, Huang S, de Pasquale R, McGehrin K, Lee HK, Zhao K, Kirkwood A (2012) Dark exposure extends the integration window for spike-timingdependent plasticity. J Neurosci 32:15027-15035. CrossRef Medline

Hardingham N, Wright N, Dachtler J, Fox K (2008) Sensory deprivation unmasks a PKA-dependent synaptic plasticity mechanism that operates in parallel with CaMKII. Neuron 60:861-874. CrossRef Medline

Huang S, Treviño M, He K, Ardiles A, Pasquale Rd, Guo Y, Palacios A, Huganir R, Kirkwood A (2012) Pull-push neuromodulation of LTP and LTD enables bidirectional experience-induced synaptic scaling in visual cortex. Neuron 73:497-510. CrossRef Medline

Huang S, Huganir RL, Kirkwood A (2013) Adrenergic gating of Hebbian spike-timing-dependent plasticity in cortical interneurons. J Neurosci 32: 13171-13178. CrossRef Medline

Huganir RL, Nicoll RA (2013) AMPARs and synaptic plasticity: the last 25 years. Neuron 80:704-717. CrossRef Medline

Jiang B, Huang ZJ, Morales B, Kirkwood A (2005) Maturation of GABAergic transmission and the timing of plasticity in visual cortex. Brain Res Brain Res Rev 50:126-133. CrossRef Medline

Jiang B, Treviño M, Kirkwood A (2007) Sequential development of longterm potentiation and depression in different layers of the mouse visual cortex. J Neurosci 27:9648-9652. CrossRef Medline

Kirkwood A, Rozas C, Kirkwood J, Perez F, Bear MF (1999) Modulation of long-term depression in visual cortex by acetylcholine and norepinephrine. J Neurosci 19:1599-1609. Medline

Koch G (2013) Do studies on cortical plasticity provide a rationale for using non-invasive brain stimulation as a treatment for Parkinson's disease patients? Front Neurol 4:180. Medline

Lisman J, Spruston N (2005) Postsynaptic depolarization requirements for LTP and LTD: a critique of spike timing-dependent plasticity. Nat Neurosci 8:839-841. CrossRef Medline

Massey PV, Bhabra G, Cho K, Brown MW, Bashir ZI (2001) Activation of muscarinic receptors induces protein synthesis-dependent long-lasting depression in the perirhinal cortex. Eur J Neurosci 14:145-152. CrossRef Medline

McCoy P, Norton TT, McMahon LL (2008) Layer 2/3 synapses in monocular and binocular regions of tree shrew visual cortex express $\mathrm{mAChR}$-dependent long-term depression and long-term potentiation. J Neurophysiol 100:336345. CrossRef Medline

Richards BA, Aizenman CD, Akerman CJ (2010) In vivo spike-timingdependent plasticity in the optic tectum of Xenopus laevis. Front Synaptic Neurosci 2:7. CrossRef Medline

Schulz R, Gerloff C, Hummel FC (2013) Non-invasive brain stimulation in neurological diseases. Neuropharmacology 64:579-587. CrossRef Medline

Seol GH, Ziburkus J, Huang S, Song L, Kim IT, Takamiya K, Huganir RL, Lee HK, Kirkwood A (2007) Neuromodulators control the polarity of spiketiming-dependent synaptic plasticity. Neuron 55:919-929. CrossRef Medline

Smith GB, Heynen AJ, Bear MF (2009) Bidirectional synaptic mechanisms of ocular dominance plasticity in visual cortex. Philos Trans R Soc Lond B Biol Sci 364:357-367. CrossRef Medline

Vallence AM, Ridding MC (2013) Non-invasive induction of plasticity in the human cortex: uses and limitations. Cortex pii: S00109452(13)00315-8. CrossRef Medline 